\title{
Functional inhibition across a visuomotor communication channel coordinates looking and reaching
}

Maureen A. Hagan ${ }^{1,2}$ and Bijan Pesaran ${ }^{2 *}$

${ }^{1}$ Biomedicine Discovery Institute, Department of Physiology Monash University

Clayton, VIC 3800

Australia

${ }^{2}$ Center for Neural Science

New York University

New York, NY 10003

*Correspondence to: bijan@nyu.edu 


\begin{abstract}
Understanding how natural behaviors are controlled depends on understanding the neural mechanisms of multiregional communication. Eye-hand coordination, a natural behavior shared by primates, is controlled by the posterior parietal cortex (PPC), a brain structure that expanded substantially in primate evolution. Here, we show that neurons within the saccade and reach regions within PPC communicate over a visuomotor channel to coordinate looking and reaching. During gaze-anchoring behavior, when saccades are transiently-inhibited by coordinated reaches, PPC neuron firing rates covary with beta-frequency $(15-25 \mathrm{~Hz})$ neuronal coherence. Decreases in parietal saccade neuron spiking correlated with gaze-anchoring behavior when the channel was "open" and not "closed". Functional inhibition across beta-frequency-coherent communication channels may be a general mechanism for flexibly coordinating our natural behavior.
\end{abstract}

One Sentence Summary Inhibitory communication through a visuomotor channel mediates the coordination of eye and hand movements. 


\section{Main text}

The primate visual system contains multiple information processing channels that transform and transmit object-based information, such as identity, shape, color, form, location, and motion (1). The visual cortices project to the posterior parietal and frontal cortices which expanded substantially in primate evolution $(2,3)$ and further transform visual information according to the goals of movement, in visuomotor channels that ultimately generate motor commands $(4,5)$. In each case, channels support communication between distinct groups of neurons in an anatomical pathway. Communication channels may also flexibly integrate information across sensory and motor pathways to support more complex behaviors (6). Eyehand coordination, which combines binocular, foveal vision and manual dexterity in a flexible, natural behavior, provides an excellent example. We coordinate saccadic eye movements to foveate targets and make accurate reach-and-grasp movements $(7,8)$. The parietal reach and saccade regions are necessary for coordinated behavior $(4,5)$ and are anatomically separate yet interconnected (9). Consequently, a visuomotor communication channel may support multiregional communication to coordinate looking and reaching.

Here we investigate the temporal coordination of looking and reaching in a behavioral coordination strategy called gaze-anchoring, originally demonstrated in humans $(10,11)$. In gaze-anchoring, during and shortly after coordinated look-and-reach movements, saccades to newly-presented targets have longer latencies, as if gaze is anchored to the target of the ongoing reach. Gaze-anchoring momentarily extends foveal vision around coordinated movements and improves movement accuracy by allowing coordinated reaches to inhibit upcoming saccades. We hypothesized that multiregional communication over a communication channel allows neurons in the parietal reach system to inhibit neurons in the parietal saccade system in order to transiently inhibit eye movements to newly-presented visual targets and improve the accuracy of an ongoing coordinated reach (Fig 1A). 
We trained two adult macaque monkeys to perform a behavioral task that naturallyelicited gaze-anchoring (Fig 1B). On coordinated-movement trials, each monkey made a reach and saccade followed by a second saccade (RS task). On individual-movement trials, each monkey made two saccades and no reaches (SS task). In the RS task, we presented the second saccade target after the reach (0-800 ms after reach completion). We then matched the time interval from the first saccade to the second saccade target onset across the SS and RS tasks. Inhibition of saccades to newly-presented targets was revealed by comparing the secondsaccade reaction times (SSRTs) between the RS and SS tasks. As in humans, both monkeys exhibited gaze-anchoring naturally without behavioral training (Materials and Methods).

SSRTs were significantly longer only when the second target appeared within $300 \mathrm{~ms}$ of the reach (peri-reach trials, Fig 1C, M1: $p=2 \times 10^{-277}, M 2: p=6 \times 10^{-60}$, rank-sum test, compared to SS task). On peri-reach trials, reach accuracy improved with longer SSRTs (Fig 1D, light green, $M 1: R=-0.33$, slope=-17.8 ms/deg, $p=1 \times 10^{-95}, M 2: R=-0.15$, slope=-7.5 ms/deg, $p=2 \times 10^{-33}$, Pearson pairwise linear correlation), but not on post-reach trials (target onset $500-800$ ms after reach completion; Fig 1D, dark green, $M 1: R=-0.069$, slope=-2.3 ms/deg, $p=2 \times 10^{-4}, M 2$ : $\mathrm{R}=0.061$, slope $=1.5 \mathrm{~ms} / \mathrm{deg}, \mathrm{p}=6 \times 10^{-5}$, Pearson pairwise linear correlation). Longer SSRTs did not reflect a generic dual-task interference effect as they correlated with reach accuracy and were directly tied to coordinated behavior (Supplementary Text, Fig S1). Thus, gaze-anchoring occurs briefly around the reach and involves trial-by-trial changes in reach and saccade movements consistent with look-reach communication.

To investigate neural mechanisms of gaze-anchoring, we recorded from 120 spatiallyselective neurons in the parietal reach system (parietal reach region, $P R R, 34$ neurons) and the parietal saccade system (lateral intraparietal area, area LIP, 86 neurons; Fig S2, Methods). We 
presented the first movement target in the response field of a PRR neuron and the second target in the response field of an area LIP neuron (Materials and Methods). Consistent with a role in guiding the reach, PRR neurons fired more during coordinated reaches, but not during saccades made alone (Fig 1E). Consistent with a role in guiding the saccade, area LIP neurons fired more before the saccade to the second saccade target (Fig 1F). Importantly, area LIP activity was transiently suppressed during RS trials around the time of the reach (Fig 1F, move vs pre-move: median fractional difference $(M F D)=-0.18, p=0.01$, sign-rank test) and no change was observed on SS trials (MFD=0.01, $\mathrm{p}=0.10$, sign-rank test). Comparing RS and $S S$ trials, PRR neurons fired significantly more around the coordinated reach (peri-reach: Fig 1G, MFD $=0.49, p=3.7 \times 10^{-4}$, sign-rank test) and area LIP neurons fired significantly less (Fig 1I, MFD=-0.11, $p=0.001$, sign-rank test). Firing rates did not differ $500 \mathrm{~ms}$ after the reach (postreach: PRR, Fig 1G, MFD=-0.17, p=0.24; Area LIP, Fig 1I, MFD=-0.04, p=0.51, sign-rank test).

Trial-by-trial changes in area LIP and PRR firing also revealed neural gaze-anchoring effects. On peri-reach RS trials, PRR neurons fired more on trials with longer SSRTs (Fig 1H, $\mathrm{R}=0.11$, slope $=0.44 \mathrm{~ms} /\left(\mathrm{sp} \cdot \mathrm{s}^{-1}\right), \mathrm{p}=0.001$, Pearson pairwise linear correlation), and area LIP neurons fired more before shorter SSRTs (Fig 1J, R =-0.10, slope=-0.37 ms/(sp $\cdot \mathrm{s}^{-1}$ ), $p=6.67 \times 10^{-7}$, Pearson pairwise linear correlation). Neural gaze-anchoring effects were specific to coordinated movements and were not observed on SS trials (PRR: $R=-0.12$, slope $=-0.31$ $\mathrm{ms} /\left(\mathrm{sp} \cdot \mathrm{s}^{-1}\right), \mathrm{p}=0.004$. Area LIP: $\mathrm{R}=-0.02, \mathrm{p}=0.54$, Pearson pairwise linear correlation) or on postreach trials $(P R R: R=-0.06, p=0.35$. Area LIP: $R=-0.03, p=0.29$, Pearson pairwise linear correlation).

To test the hypothesis that a communication channel coordinates looking and reaching, we analyzed neural activity to measure multiregional communication. Coherent neural dynamics measure functional interactions between groups of neurons by measuring fluctuations in neural 
excitability. Changes in excitability lead to increases in spiking at particular times or phases of coherent activity and may support multiregional communication. In the reach and saccade systems, neuronal coherence in the beta-frequency band $(\sim 12-30 \mathrm{~Hz})$ is particularly prominent, predicts upcoming movements (12-18), encodes movement choice (19,20), decreases around movement initiation $(12,21-24)$, reflects the coordination of sensorimotor activity $(19,21,25-$ $29)$, top-down feedback $(25,30,31)$ and multiregional integration (32). This suggests that the phase of beta-frequency neuronal coherence may reflect neural excitability and support multiregional communication between the reach and saccade systems.

We analyzed data from 151 experimental sessions with PRR spiking recorded simultaneously with local field potentials (LFP) in both PRR and area LIP. LFP activity in area LIP and PRR transiently synchronized with packets of activity occurring in-phase (Fig 2A, arrow) and out-of-phase (Fig 2B, arrow). PRR spiking was coherent with simultaneouslyrecorded beta-frequency LFPs in each area (Fig S3). To characterize coherent spiking trial-bytrial, we calculated the beta-frequency phase of LFP activity in both area LIP and PRR at times when PRR neurons spiked and averaged across spiking events during each task epoch. During the peri-reach epoch of the RS task, when behavior revealed look-reach communication, PRR spiking tended to occur when LFPs in both areas were in-phase (Example: Fig 2C. Population: Fig S3Ai, mean $\pm S D$ phase: $P R R=132 \pm 74^{\circ} p=6 \times 10^{-57} ;$ LIP $=173 \pm 79^{\circ}, p=2 \times 10^{-6}$, Rayleigh's test, Materials and Methods). During the SS task before the presentation of the second target, when behavior showed no communication, PRR spiking tended to occur when LFPs were at other phases (Example: Fig 2D. Population: S3Aiii, mean \pm SD phase: $P R R=127 \pm 74^{\circ}, p=3 \times 10^{-}$ ${ }^{41}$; LIP $=53 \pm 78^{\circ}, p=2 \times 10^{-10}$, Rayleigh's test). Therefore, dual-coherent phase, the difference between the LFP phase in the reach and saccade systems aligned to reach neurons spiking (Fig $2 \mathrm{Ci}, \mathrm{ii}, \mathrm{Di}, \mathrm{ii}$ ), changed between trials which required differences in reach-saccade communication. 
To test whether neural coherence reflected multiregional communication, we measured how well dual coherence predicted changes in coordinated behaviour. On peri-reach trials, SSRT varied trial-by-trial with dual-coherent phase (Fig 2E, $p=2 \times 10^{-11}$, von Mises test, Materials and Methods). SSRTs were slowest, $\sim 196 \mathrm{~ms}$, on trials with dual-coherent phase of $\sim 76^{\circ}$ (mean: $76,95 \% \mathrm{Cl}[60,92]$ von Mises fit) and fastest, $183 \mathrm{~ms}$, on trials with dual-coherent phase of $\sim 90^{\circ}$ (SSRT peri-reach vs saccade trials centered at $68^{\circ} p=1 \times 10^{-4}$, permutation test). On trials with other dual-coherent phases, SSRT did not increase (SSRT peri-reach vs saccade trials centered at $-112^{\circ}, p=0.62$, permutation test). On trials with no expected multiregional communication, dual-coherent phase and SSRT did not significantly covary (post-reach: $p=1$, SS task: $p=0.44$, von Mises test).

Examining gaze-anchoring effects and PRR firing with respect to LIP phase and PRR phase separately reinforced the importance of PRR dual-coherent phase on peri-reach trials (Fig 2Fi, dashed line corresponding dual-coherent phase shown by Fig 2E, triangle). Importantly, PRR neuron firing with respect to LIP phase alone did not predict multiregional communication because gaze-anchoring effects did not depend on LIP-only phase at any time (peri-reach: $p=1$; post-reach: $p=1$; SS task: $p=0.82$, von Mises test). Interestingly, PRR-only phase revealed gaze-anchoring effects on peri-reach trials (mean $=-126^{\circ} 95 \% \mathrm{Cl}[-153,-99]$, $p=2 \times 10^{-3}$, von Mises test $)$ and not on post-reach trials $(p=1$, von Mises test) or SS task trials $(p=1$, von Mises test). However, the dual-coherent phase model predicted SSRT better than the PRR-only phase model and with a smaller expected generalization error (Supplementary Text).

Dual-coherent phase also best predicted reach accuracy. Reaches were most accurate on peri-reach trials when PRR neurons fired with around $85^{\circ}$ dual-coherent phase difference 
(Fig 2G, mean $=-96^{\circ}, 95 \% \mathrm{Cl}[-112,-80], p=3 \times 10^{-12}$, von Mises test) but not on post reach trials ( $p=1$, von Mises test). Improved accuracy on peri-reach trials was specific to dual-coherent phase (Fig 2G, triangle; Fig 2Hi, dashed line). Accuracy did not depend on PRR phase alone (peri-reach: $p=1$, von Mises test) or LIP phase alone (peri-reach: $p=0.06$; post-reach: $p=1$, von Mises test), but was weakly modulated by PRR phase alone on post-reach trials (mean $=-163^{\circ}$, $95 \% \mathrm{Cl}[-200,-126], p=0.01$, von Mises test). As for SSRT, the fitted dual-coherent phase model predicted peri-reach reach accuracy with the greatest log-likelihood and smallest expected generalization error (Supplementary Text). These results are not simply due to changes in firing rates of neurons in each area, and additional analyses supported the conclusion that gaze-anchoring neural effects are specific to beta-frequency neural coherence (Supplementary Text, Figs S3-5).

On peri-reach trials, area LIP neurons fire more before shorter SSRTs (Fig 1J) and SSRT varies trial-by-trial with dual-coherent phase (Fig 2E). If area LIP firing reflects multiregional communication, beta-frequency neural coherence should modulate the state of a communication channel that links the parietal reach and saccade regions, in a channel-state model (Fig 3A). Analyzing the firing of LIP neurons, SSRT and PRR dual-coherent phase revealed that area LIP firing rate was consistent with the channel-state model. Area LIP firing significantly covaried with PRR dual-coherent phase during gaze-anchoring (Fig 3B, peri-reach $p=0$, von Mises test, 32 spatially-selective PRR-LIP neuron pairs; 88 spike-spike-field-field sessions also featuring simultaneous PRR-LIP LFP recordings; Materials and Methods) but not post-reach ( $p=1$, von Mises test) or saccade-alone trials ( $p=1$, von Mises test). Importantly, area LIP neuron firing rates were maximally suppressed at the same preferred phase angle as the SSRT variations with PRR dual-coherent phase (minimum firing rate at 92 deg, mean=-88 95\% CI [-97, -79], von Mises fit). This demonstrates that inhibited area LIP activity is associated 
with increased SSRT during gaze-anchoring and may be mediated by a state-dependent communication channel in which area LIP receives input from the reach system.

Unlike area LIP activity, the overall rate of PRR spiking did not depend on dual-coherent channel state. PRR neurons fired the same number of spikes across trials independent of dualcoherent phase during gaze-anchoring (Fig 3C, $p=1$, von Mises test). Interestingly, PRR firing rate also weakly covaried with dual-coherent state during post-reach ( $p=0.04$, von Mises test) and saccade-alone trials ( $p=5 \times 10^{-14}$, von Mises test). This is also consistent with the channelstate model as overall PRR firing should not depend on channel state.

Nevertheless, the channel-state model predicts that PRR firing should support a sender role in a communication. Sender-reach activity should be communicated, and influence the receiver-saccade, when dual-coherent phase is preferred, "channel open state", and not when dual-coherent phase is less-preferred, "channel closed state". If so, increases in PRR-sender spikes at times with preferred-relative-phase should be associated with longer SSRTs and inhibited LIP-receiver spiking. We therefore tested the channel-state model by modeling the dependency between SSRT and PRR firing and asking whether SSRT depends on input drive from PRR that is gain-modulated by channel state, e.g. dual coherent phase.

PRR firing covaried with gaze-anchoring-related increases in SSRT specifically on trials with preferred dual-coherent phase, the channel open state (Fig 3Di, R=0.11, $p=5 \times 10^{-3}$, Pearson pairwise linear correlation). SSRT did not change when the PRR dual-coherent phase was non-preferred even when PRR-sender activity increased ten-fold to $30 \mathrm{sp} / \mathrm{s}$ (Fig 3Di). Covariation of combined PRR spiking and channel state with SSRT was specific to gazeanchoring and not present during post-reach trials (Fig 3Dii, at preferred phase $\mathrm{R}=0.04, p=0.54$, 
Pearson pairwise linear correlation) and saccades made alone (Fig 3Diii, at preferred phase: $\mathrm{R}=-0.03, \mathrm{p}=0.47$, Pearson pairwise linear correlation).

These results demonstrate that gaze-anchoring-related increases in PRR and area LIP firing fulfill sender and receiver roles in a communication channel. The overall level of PRR firing is not modulated by channel state, consistent with a sender. The level of area LIP firing is modulated by channel state, consistent with a receiver. The relationships between PRR firing, LIP firing and SSRT in the channel-state model further demonstrate that reach-saccade interactions are not static and are dynamic, and are consistent with channel state that depends on dual-coherent phase. For simplicity we refer to discrete "open/closed" channel state, but the underlying mechanism need not be discrete, and our results are consistent with a continuouslyvarying channel gain. i.e. "Open/closed channel state" could be stated as "high/low channel gain".

Overall, our results demonstrate how a visuomotor communication channel linking the parietal saccade and reach systems can orchestrate the temporal coordination of where we look and reach. We use gaze-anchoring to track directional communication from the reach system to the saccade system. Area LIP activity related to the second saccade could not be responsible for signals in PRR that improve reach accuracy, because we presented the second saccade target after the reach, and area LIP neural response fields for the first and second saccades did not overlap in space. Gaze-anchoring also features a naturally-expressed behavior in which two behavioral outputs depend on each other. Consequently, gaze-anchoring reveals communication behaviorally without the confounding influence of behavioral over-training that could alter the underlying mechanisms of multiregional communication. 
Inhibitory coordinated control mechanisms have been implicated in other coordinated behaviors such as when making oblique saccades (33), looking and reaching (22, 34), and reaching and grasping (35). Our results demonstrate that inhibitory coordinated control plays a role in mechanisms of visual-spatial cognition more broadly. We show that saccades to visual targets presented unexpectedly at different spatial locations are initiated more slowly, consistent with suppressed target selection throughout area LIP.

Beta-frequency coherence may support other behavioral processes requiring multiregional functional inhibition (36). Previous work has suggested that propagating beta waves in V4 influence the processing of behaviorally-relevant stimuli (37) and thalamocortical beta oscillations have also been implicated in inhibition $(38-40)$ and slowing $(41,42)$ of movement initiation. Beta-frequency activity facilitates ongoing behaviors $(26,42)$, potentially at the expense of upcoming (43-45), and beta coherence has been linked to GABAergic activity both experimentally (46-49) and through modelling (46). Beta-frequency coherence generation may specifically reflect synaptic influences on inhibitory neurons. Finally, we show that parietal gamma-band coherence is not involved in gaze-anchoring-related functional inhibition, consistent with a proposed role for gamma-band coherence in other processes, such as feedforward excitation (30).

How is the communication channel controlled? LIP and PRR could coordinate their activity to control the channel, and we do not rule out a role for feedback processing from area LIP to PRR (30). However, dual coherent beta-band modulation to "open" and "close" the channel may not be strictly consistent with either feedforward or feedback inhibition between area LIP and PRR. Dual coherence may also reflect the action of network modulators and shared inputs to both area LIP and PRR. According to this modulator view, the communication channel may be controlled by a distributed network of neurons spanning other brain regions 
involved in the organization of behavior - such as prefrontal, medial frontal cortex as well as subcortical regions such as the basal ganglia and thalamus. Around coordinated movements, modulations of coherent neuronal dynamics transiently open a communication channel between the parietal reach and saccade regions, allowing reach neurons to suppress target selection and anchor gaze just long enough to improve movement accuracy.

In conclusion, our results show that functional inhibition across beta-coherent communication channels may be a general mechanism for flexibly coordinating our natural behavior with important implications for understanding the brain mechanisms of multiregional communication. 


\section{References and Notes:}

1. D. Milner, M. Goodale, The Visual Brain in Action (Oxford University Press, USA, ed. 2, 2006).

2. J. H. Kaas, O. A. Gharbawie, I. Stepniewska, The organization and evolution of dorsal stream multisensory motor pathways in primates. Front. Neuroanat. 5, 34 (2011).

3. T. A. Chaplin, H.-H. Yu, J. G. M. Soares, R. Gattass, M. G. P. Rosa, A conserved pattern of differential expansion of cortical areas in simian primates. J. Neurosci. 33, 15120-15125 (2013).

4. V. Gaveau, D. Pélisson, A. Blangero, C. Urquizar, C. Prablanc, A. Vighetto, L. Pisella, Saccade control and eye-hand coordination in optic ataxia. Neuropsychologia. 46, 475-486 (2008).

5. L. Pisella, L. Sergio, A. Blangero, H. Torchin, A. Vighetto, Y. Rossetti, Optic ataxia and the function of the dorsal stream: contributions to perception and action. Neuropsychologia. 47, 3033-3044 (2009).

6. G. Hahn, A. Ponce-Alvarez, G. Deco, A. Aertsen, A. Kumar, Portraits of communication in neuronal networks. Nat. Rev. Neurosci. 20, 117-127 (2019).

7. R. A. Abrams, D. E. Meyer, S. Kornblum, Eye-hand coordination: oculomotor control in rapid aimed limb movements. J. Exp. Psychol. Hum. Percept. Perform. 16, 248-267 (1990).

8. W. F. Helsen, D. Elliott, J. L. Starkes, K. L. Ricker, Coupling of eye, finger, elbow, and shoulder movements during manual aiming. J. Mot. Behav. 32, 241-248 (2000).

9. J. W. Lewis, D. C. van Essen, Corticocortical connections of visual, sensorimotor, and multimodal processing areas in the parietal lobe of the macaque monkey. J. Comp. Neurol. 428, 112-137 (2000).

10. S. F. Neggers, H. Bekkering, Ocular gaze is anchored to the target of an ongoing pointing movement. J. Neurophysiol. 83, 639-651 (2000).

11. S. F. Neggers, H. Bekkering, Gaze anchoring to a pointing target is present during the entire pointing movement and is driven by a non-visual signal. J. Neurophysiol. 86, 961970 (2001).

12. A.-A. Pape, M. Siegel, Motor cortex activity predicts response alternation during sensorimotor decisions. Nature Communications. 7 (2016), , doi:10.1038/ncomms13098.

13. H. Scherberger, M. R. Jarvis, R. A. Andersen, Cortical Local Field Potential Encodes Movement Intentions in the Posterior Parietal Cortex. Neuron. 46 (2005), pp. 347-354.

14. R. L. Spinks, A. Kraskov, T. Brochier, M. A. Umilta, R. N. Lemon, Selectivity for Grasp in Local Field Potential and Single Neuron Activity Recorded Simultaneously from M1 and F5 in the Awake Macaque Monkey. Journal of Neuroscience. 28 (2008), pp. 10961-10971.

15. J. Reimer, N. G. Hatsopoulos, Periodicity and Evoked Responses in Motor Cortex. Journal of Neuroscience. 30 (2010), pp. 11506-11515. 
16. M. Saleh, J. Reimer, R. Penn, C. L. Ojakangas, N. G. Hatsopoulos, Fast and Slow Oscillations in Human Primary Motor Cortex Predict Oncoming Behaviorally Relevant Cues. Neuron. 65 (2010), pp. 461-471.

17. E. J. Hwang, R. A. Andersen, Effects of visual stimulation on LFPs, spikes, and LFP-spike relations in PRR. Journal of Neurophysiology. 105 (2011), pp. 1850-1860.

18. B. E. Kilavik, A. Ponce-Alvarez, R. Trachel, J. Confais, S. Takerkart, A. Riehle, ContextRelated Frequency Modulations of Macaque Motor Cortical LFP Beta Oscillations. Cerebral Cortex. 22 (2012), pp. 2148-2159.

19. S. Babapoor-Farrokhran, M. Vinck, T. Womelsdorf, S. Everling, Theta and beta synchrony coordinate frontal eye fields and anterior cingulate cortex during sensorimotor mapping. Nature Communications. 8 (2017), , doi:10.1038/ncomms13967.

20. Y. T. Wong, M. M. Fabiszak, Y. Novikov, N. D. Daw, B. Pesaran, Coherent neuronal ensembles are rapidly recruited when making a look-reach decision. Nature Neuroscience. 19 (2016), pp. 327-334.

21. J. W. Chung, E. Ofori, G. Misra, C. W. Hess, D. E. Vaillancourt, Beta-band activity and connectivity in sensorimotor and parietal cortex are important for accurate motor performance. Neurolmage. 144 (2017), pp. 164-173.

22. H. L. Dean, M. A. Hagan, B. Pesaran, Only Coherent Spiking in Posterior Parietal Cortex Coordinates Looking and Reaching. Neuron. 73 (2012), pp. 829-841.

23. W. P. Medendorp, G. F. I. Kramer, O. Jensen, R. Oostenveld, J. -M. Schoffelen, P. Fries, Oscillatory Activity in Human Parietal and Occipital Cortex Shows Hemispheric Lateralization and Memory Effects in a Delayed Double-Step Saccade Task. Cerebral Cortex. 17 (2007), pp. 2364-2374.

24. M. A. Hagan, H. L. Dean, B. Pesaran, Spike-field activity in parietal area LIP during coordinated reach and saccade movements. Journal of Neurophysiology. 107 (2012), pp. 1275-1290.

25. A. Brovelli, M. Ding, A. Ledberg, Y. Chen, R. Nakamura, S. L. Bressler, Beta oscillations in a large-scale sensorimotor cortical network: Directional influences revealed by Granger causality. Proceedings of the National Academy of Sciences. 101 (2004), pp. 9849-9854.

26. R. Kristeva, L. Patino, W. Omlor, Beta-range cortical motor spectral power and corticomuscular coherence as a mechanism for effective corticospinal interaction during steady-state motor output. Neurolmage. 36 (2007), pp. 785-792.

27. T. H. Donner, M. Siegel, A framework for local cortical oscillation patterns. Trends in Cognitive Sciences. 15 (2011), pp. 191-199.

28. V. Romei, M. Bauer, J. L. Brooks, M. Economides, W. Penny, G. Thut, J. Driver, S. Bestmann, Causal evidence that intrinsic beta-frequency is relevant for enhanced signal propagation in the motor system as shown through rhythmic TMS. Neurolmage. 126 (2016), pp. 120-130.

29. D. J. Hawellek, Y. T. Wong, B. Pesaran, Temporal coding of reward-guided choice in the 
posterior parietal cortex. Proceedings of the National Academy of Sciences. 113 (2016), pp. 13492-13497.

30. A. M. Bastos, J. Vezoli, C. A. Bosman, J.-M. Schoffelen, R. Oostenveld, J. R. Dowdall, P. De Weerd, H. Kennedy, P. Fries, Visual Areas Exert Feedforward and Feedback Influences through Distinct Frequency Channels. Neuron. 85 (2015), pp. 390-401.

31. J. H. Lee, M. A. Whittington, N. J. Kopell, Top-Down Beta Rhythms Support Selective Attention via Interlaminar Interaction: A Model. PLoS Computational Biology. 9 (2013), p. e1003164.

32. H. Tan, C. Wade, P. Brown, Post-Movement Beta Activity in Sensorimotor Cortex Indexes Confidence in the Estimations from Internal Models. The Journal of Neuroscience. 36 (2016), pp. 1516-1528.

33. A. F. Fuchs, C. R. Kaneko, C. A. Scudder, Brainstem control of saccadic eye movements. Annu. Rev. Neurosci. 8, 307-337 (1985).

34. L. Rogal, B. Fischer, Eye-hand-coordination: a model for computing reaction times in a visually guided reach task. Biol. Cybern. 55, 263-273 (1986).

35. B. Hoff, M. A. Arbib, Models of Trajectory Formation and Temporal Interaction of Reach and Grasp. J. Mot. Behav. 25, 175-192 (1993).

36. Y. Zhang, Y. Chen, S. L. Bressler, M. Ding, Response preparation and inhibition: The role of the cortical sensorimotor beta rhythm. Neuroscience. 156 (2008), pp. 238-246.

37. T. P. Zanos, P. J. Mineault, K. T. Nasiotis, D. Guitton, C. C. Pack, A sensorimotor role for traveling waves in primate visual cortex. Neuron. 85, 615-627 (2015).

38. A. A. Kühn, D. Williams, A. Kupsch, P. Limousin, M. Hariz, G. Schneider, K. Yarrow, P. Brown, Event-related beta desynchronization in human subthalamic nucleus correlates with motor performance. Brain. 127 (2004), pp. 735-746.

39. B. Zavala, S. Damera, J. W. Dong, C. Lungu, P. Brown, K. A. Zaghloul, Human Subthalamic Nucleus Theta and Beta Oscillations Entrain Neuronal Firing During Sensorimotor Conflict. Cerebral Cortex (2015), p. bhv244.

40. N. Swann, H. Poizner, M. Houser, S. Gould, I. Greenhouse, W. Cai, J. Strunk, J. George, A. R. Aron, Deep Brain Stimulation of the Subthalamic Nucleus Alters the Cortical Profile of Response Inhibition in the Beta Frequency Band: A Scalp EEG Study in Parkinson's Disease. Journal of Neuroscience. 31 (2011), pp. 5721-5729.

41. A. Pogosyan, L. D. Gaynor, A. Eusebio, P. Brown, Boosting Cortical Activity at Beta-Band Frequencies Slows Movement in Humans. Current Biology. 19 (2009), pp. 1637-1641.

42. A. K. Engel, P. Fries, Beta-band oscillations—signalling the status quo? Current Opinion in Neurobiology. 20 (2010), pp. 156-165.

43. G. Pfurtscheller, A. Stancák, C. Neuper, Post-movement beta synchronization. A correlate of an idling motor area? Electroencephalography and Clinical Neurophysiology. 98 (1996), pp. 281-293. 
44. T. Gilbertson, E. Lalo, L. Doyle, V. Di Lazzaro, B. Cioni, P. Brown, Existing motor state is favored at the expense of new movement during 13-35 Hz oscillatory synchrony in the human corticospinal system. J. Neurosci. 25, 7771-7779 (2005).

45. B. Pastötter, S. Hanslmayr, K.-H. Bäuml, Inhibition of Return Arises from Inhibition of Response Processes: An Analysis of Oscillatory Beta Activity. Journal of Cognitive Neuroscience. 20 (2008), pp. 65-75.

46. O. Jensen, P. Goel, N. Kopell, M. Pohja, R. Hari, B. Ermentrout, On the human sensorimotor-cortex beta rhythm: Sources and modeling. Neurolmage. 26 (2005), pp. 347355.

47. N. Yamawaki, I. M. Stanford, S. D. Hall, G. L. Woodhall, Pharmacologically induced and stimulus evoked rhythmic neuronal oscillatory activity in the primary motor cortex in vitro. Neuroscience. 151 (2008), pp. 386-395.

48. S. D. Hall, I. M. Stanford, N. Yamawaki, C. J. McAllister, K. C. Rönnqvist, G. L. Woodhall, P. L. Furlong, The role of GABAergic modulation in motor function related neuronal network activity. Neurolmage. 56 (2011), pp. 1506-1510.

49. T. J. Baumgarten, G. Oeltzschner, N. Hoogenboom, H.-J. Wittsack, A. Schnitzler, J. Lange, Beta Peak Frequencies at Rest Correlate with Endogenous GABA / Cr Concentrations in Sensorimotor Cortex Areas. PLOS ONE. 11 (2016), p. e0156829. 


\section{Acknowledgements}

We would like to thank Gerardo Moreno for surgical assistance, Roch Comeau, Stephen Frey and Brian Hynes for custom modifications to the BrainSight system, and members of the Pesaran lab for helpful feedback. This work was supported, in part, by NIH T32 EY007136 (MAH), ARC DE180100344 (MAH), NSF CAREER Award BCS-0955701 (BP), NEI R01EY087882 (BP), the Army Research Office (BP), the Simons Foundation (BP), a McKnight Scholar Award (BP), and a Sloan Research Fellowship (BP). MAH and BP conceptualized the project and designed the experiments. MAH performed the experiments. MAH and BP analyzed the data and wrote the manuscript. The authors declare no competing interests.

\section{Supplementary Materials:}

Materials and Methods

Supplementary Text

Figures S1-S5

References (50-51) 

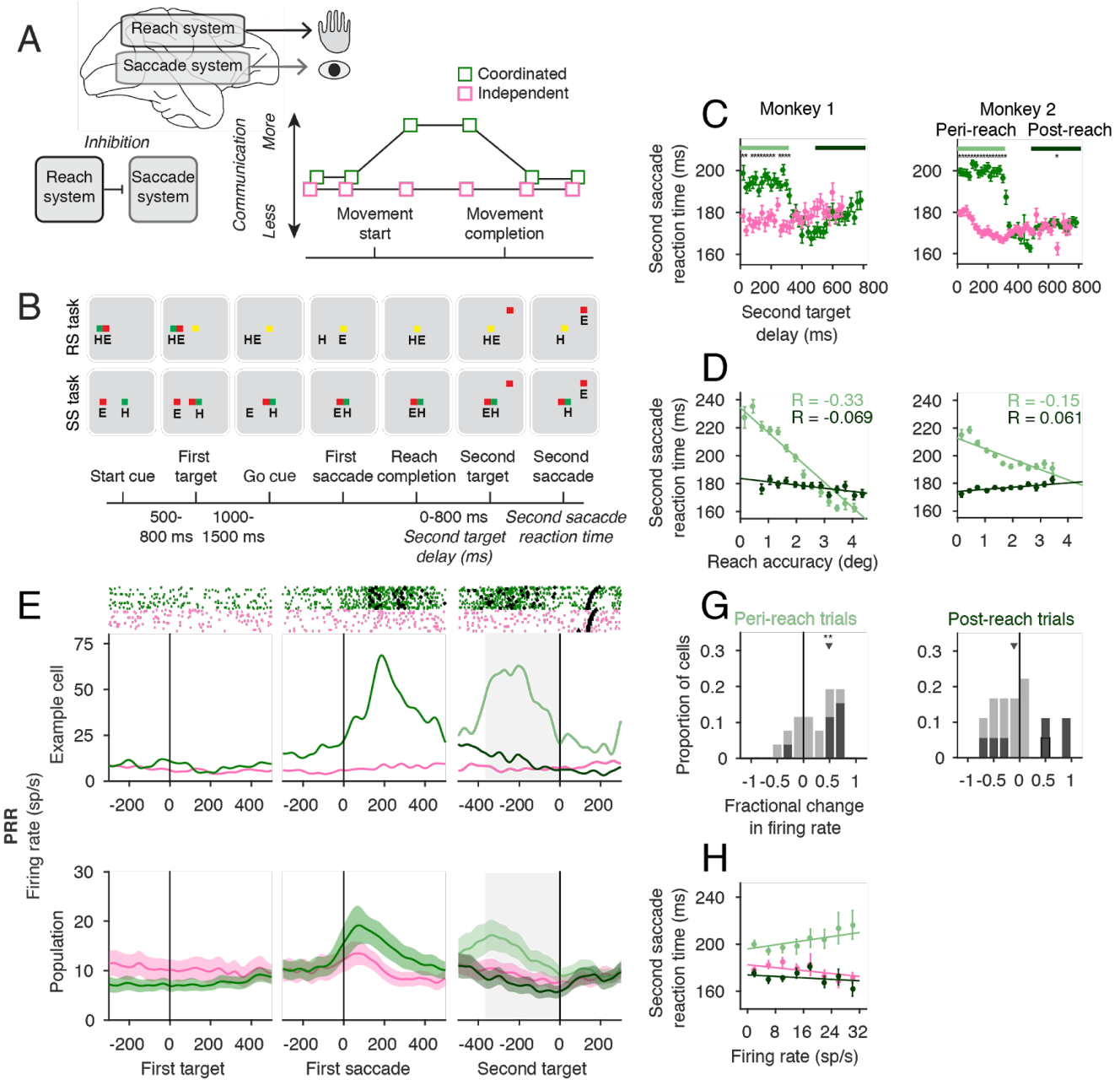

\section{$\mathrm{H}$}
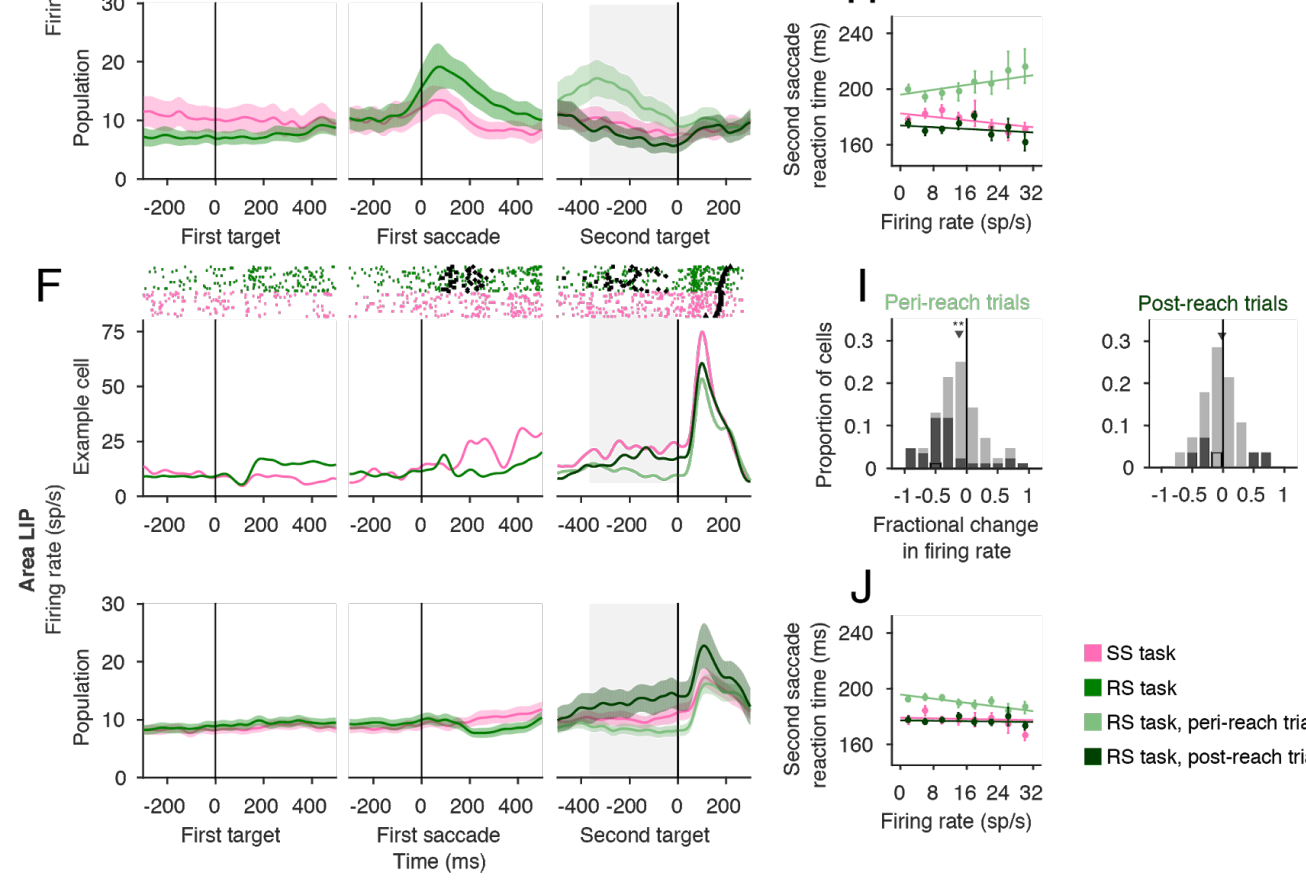

SS task
RS task
RS task, peri-reach trials
RS task, post-reach trials

Fig. 1. Coordinated behavior provides evidence of multiregional communication. (A) Schematic showing reach and saccade system communication during coordinated and independent movements. (B) Reach and saccade double-step task (RS) and Saccade doublestep task (SS), indicating hand $(\mathrm{H})$ and eye (E) position at each epoch. (C) Reaction times for 
the second saccade (SSRT) as a function of second target delay for RS task (green) and SS task (pink, stars, p < 0.05). Horizontal lines indicate peri-reach (light green) and post-reach trials (dark green). (D) SSRT as a function of reach accuracy on peri-reach (light green) and postreach (dark green) trials. (E) Example cells and population firing rates for PRR and (F) LIP . Reach start (squares), reach stop (diamond) and second saccade (triangle) are shown for each trial. Shaded area indicates trial epoch for subsequent neural analysis. (G) Fractional change in firing rate ((RS-SS)/SS) between the two tasks for PRR and (I) LIP. Cells which showed significant difference ( $p<0.05$, dark squares) and example cells (from $F, G$, black outline) are indicated. (H) Firing rates in PRR and (J) LIP on each trial type as a function of SSRT. 

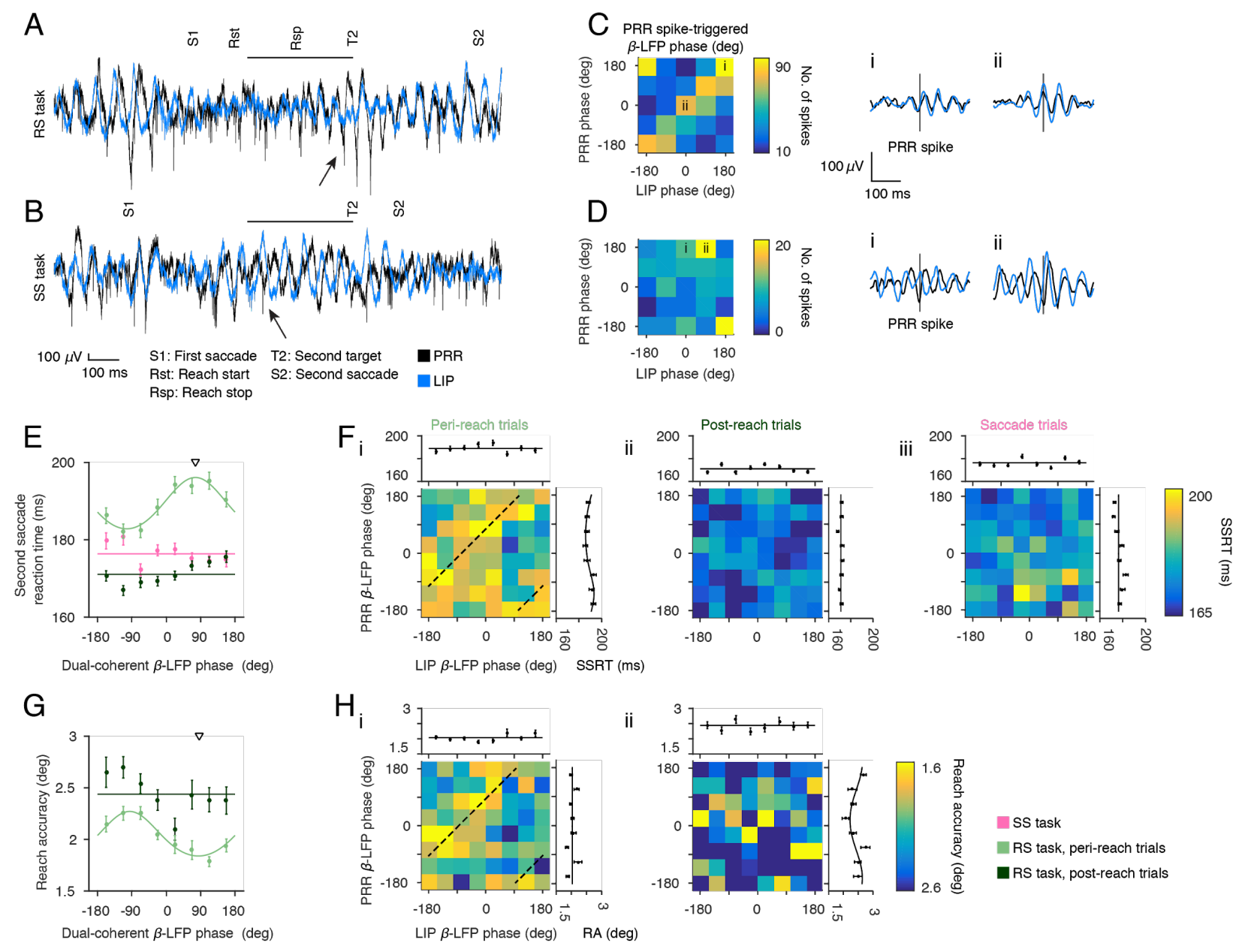

Fig. 2. Behavior depends on coherent neural dynamics. (A) Voltage traces of RS task and (B) SS task example trials (PRR: black, LIP: blue). Horizontal line indicates analysis window for neural data. Arrows indicate example spikes occurring at representative phases in each task. (C) Number of spikes (color scale) occurring in each PRR spike-triggered $\beta$ LFP phase $(20 \mathrm{~Hz})$ bin for PRR and LIP in one example cell from the RS task and (D) SS task. (E) SSRT and (G) reach accuracy as a function of dual-coherent $\beta$ LFP phase for each trial type. (F) SSRT and $(\mathbf{H})$ reach accuracy (RA, color scale) as a function of both PRR $\beta$ LFP phase and LIP $\beta$ LFP phase for each trial type. Marginals show the SSRTs as a function of phase in each area. 

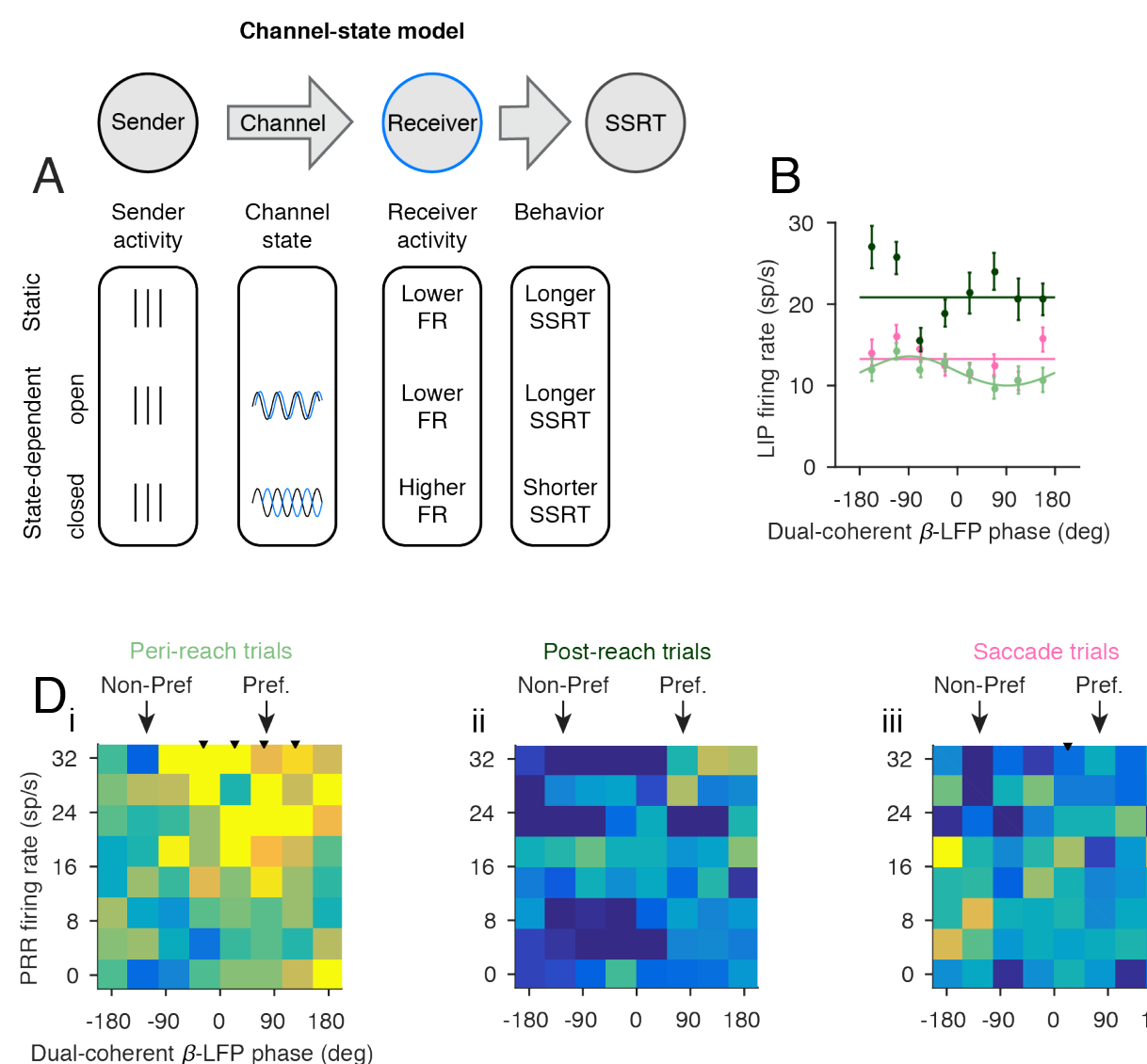
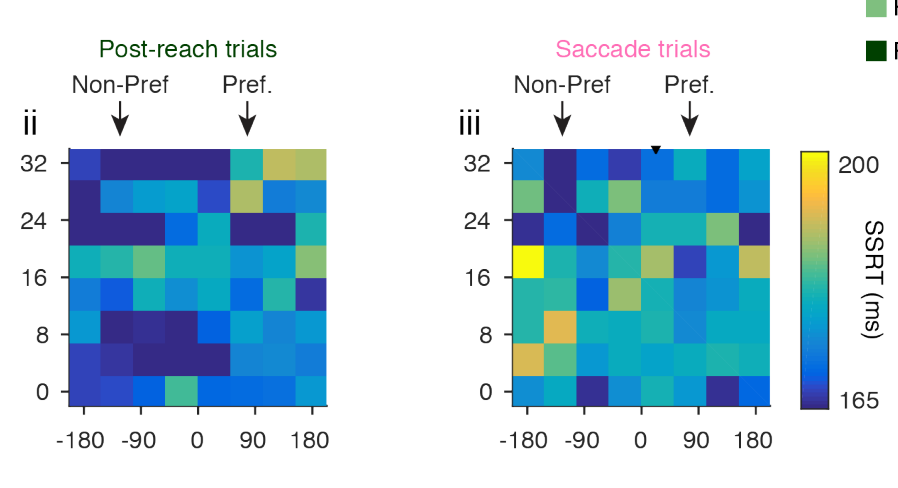

Fig. 3. State-dependent look-reach communication. (A) Channel-state model illustrating how behavior and neural activity reflect static or state-dependent sender-receiver communication channels. (B) LIP and (C) PRR firing rate and a function of dual-coherent $\beta$-LFP phase for each trial type. (D) SSRT (color scale) as a function of PRR firing rate and dual-coherent $\beta$-LFP phase for each trial type. Arrows indicate phase bins used for 'Preferred' and 'Non-preferred' channel states. Black triangles indicate columns for which the correlation with SSRT was significant $(p<0.05$, Bonferroni corrected). 